\title{
viewpoint
}

butterfly, in Ontario. Canadian Food Inspection Agency, Plant Biotechnology Office.

Trewavas, A.J. and Leaver, C.J. (2000) Conventional crops are the test of GM prejudice. Nature, 401, 640.

USDA (2000) US Agricultural Outlook (July-August 2000). USDA -ERS. http://www.agribusiness.asn.au/ NewsLinksCongressEvents/A.../21July2000USO/

Wraight, C.L., Zangeri, A.R., Carroll, M.J. and Berenbaum, M.R. (2000) Absence of toxicity of Bacillus thuringiensis pollen to black swallowtails under field conditions. Proc. Natl Acad. Sci. USA, 97, 7700-7703.

Xia, J.Y., Cui-Jin, J., Ma, L.H., Dong, S.L. and Cui, X.F. (1999) The role of transgenic Bt cotton in integrated insect pest management. Acta Gossypii Sinica, 11, 57-64.
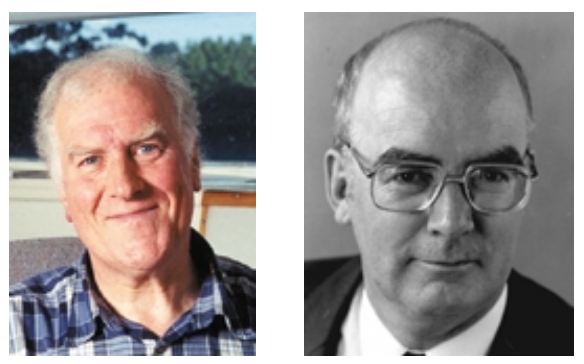

Anthony J. Trewavas is at the Institute of Cell and Molecular Biology at the University of Edinburgh and Christopher J. Leaver is at the Department of Plant Sciences at the University of Oxford.

E-mail: trewavas@srv0.bio.ed.ac.uk or chris.leaver@plant-sciences.oxford.ac.uk

DOI: 10.1093/embo-reports/kve123

\section{Foot-and-mouth disease in Europe}

\author{
FMD is economically the most important disease of farm animals. Its re-emergence in \\ Europe is likely to have consequences that go beyond severe alterations of livestock \\ production and trade $\bullet$ by Francisco Sobrino \& Esteban Domingo
}

On February 21, 2001, the United Kingdom officially declared an outbreak of foot-and-mouth disease (FMD) in England. Since then, the disease has spread like a bush fire among farm animals - mainly sheep and cattle-reaching a total of 1461 confirmed outbreaks by April 20, and, in March, appeared in continental Europe with one confirmed outbreak in The Netherlands. To prevent further spread of FMD—so far, a hopeless effort-British officials have slaughtered and destroyed more than 2 million animals, such a massive undertaking that the British army had to be ordered to help. (Updated information can be found at the Office International des Epizooties: www.oie.int).

This is the first major FMD epizootic in the UK since 1968, and it represents a monumental set-back for the non-vaccination policy that the EU implemented in 1991. Losses were initially estimated to be 6 billion Euros, but this is likely to be an underestimate. The FMD outbreak, added to the BSE crisis, must call into question whether the existing human and animal health policies in the EU are still adequate in the context of a highly competitive and a global economy.
Foot-and-mouth disease virus (FMDV), a representative of the aphthovirus genus of the Picornaviridae family, causes the disease that is ravaging the UK. The term aphthovirus derives from the Greek 'aphtha', which refers to the vesicles found in the mouth and feet of affected animals. It was first identified in 1898 by Loeffler and Frosch (Bachrach, 1968). Earlier, Fracastorius described (in a book published in 1546), a disease of cattle, which occurred in Venice in 1514, and which, most likely, was FMD. The disease was endemic in Europe from the seventeenth until the nineteenth century, and became more frequent in the first half of the twentieth century, as a result of more intensive cattle breeding and increased traffic of susceptible animals (Bachrach, 1968; Pereira, 1981). With the exception of New Zealand, FMD has occurred at one time or another in most locations of the world. An important epizootic in Mexico from 1946 until 1953 was prevented from spreading to the United States-disease-free since 1929—-through a huge surveillance programme.

The 2001 European epizootic is associated with the unprecedented entry of serotype O PanAsia FMDV from Asia (Knowles et al., 2001). The PanAsia FMDVs form a distinct phylogenetic cluster amid other type $\mathrm{O}$ viruses that have been circulating mainly in Asia and the Middle East. The virus that caused the current outbreak was first detected in 1990 in India and rapidly spread both eastward and westward. In Europe, it has found a fully susceptible animal population to thrive on.

FMDV particles are spherical, with icosahedral symmetry and devoid of a lipid envelope (Acharya et al., 1989). The capsid encloses a single-stranded RNA molecule of approximately 8500 nucleotides. After translation, proteins are processed from a single polyprotein precursor. FMDV enters the animal host through the respiratory tract or through skin abrasions, and initiates a poorly understood replication cycle with an incubation period of generally 1-8 days. A viremic phase (virus in the blood) precedes the development of the characteristic vesicles, which makes control of the virus more difficult. It has been estimated that infected cattle may harbour up to $10^{12}$ infectious units. Large amounts of virus are found in the lesions of infected animals as well as in their secretions and excretions, particularly from pigs. 


\section{viewpoint}

With its high viral load and the ability of the virus to travel through the air, infected animals are a prolific source of virus, with a very high probability of infecting other susceptible animals (Bachrach, 1968; Pereira, 1981).

Early genetic studies by Pringle and others suggested high mutation frequencies and the occurrence of RNA recombination during FMDV replication; many types of phenotypic variants were identified in these initial studies (Bachrach, 1968). Cloning and rapid nucleotide sequencing techniques documented that FMDV populations follow a quasispecies dynamics, and provided the first biochemical evidence of recombination in RNA (reviewed in Domingo et al., 2001; Sobrino et al., 2001). Similar to other RNA viruses, the error-prone nature of FMDV RNA replication stems from the absence of proof-reading processes and post-replicative repair during the viral RNA polymerisation reaction (Domingo et al., 2001). Long-term evolution of FMDV occurs through positive selection and random drift acting on essentially heterogeneous viral populations. The result is a diversification process that materialises with variable rates of mutation fixations. During persistent infections-a possible and of the virus have been described: $\mathrm{O}, \mathrm{A}, \mathrm{C}$, Asia1 and SAT1, SAT2, SAT3 (Bachrach,

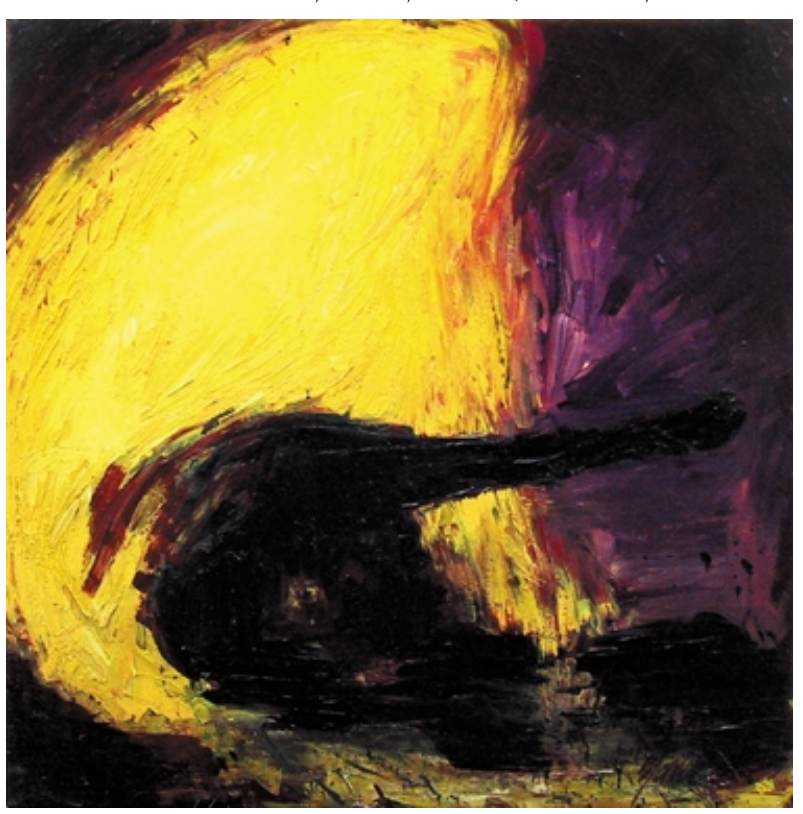

Max Benz Well Done, 1996. Oil on canvas. Courtesy of the artist

1968; Sobrino et al., 2001). The first vaccine to control FMD was used in 1953 in The Netherlands. It was based on virus grown in fragments of bovine tongue epithelium, followed by chemical treatment of virus to eliminate infectivity-the Frankel-type trivalent vaccine (Bachrach, 1968). Later, largescale production in suspensions of baby hamster kidney cells produced sufficient vaccine for extensive vaccination of cattle, which resulted in a dramatic decrease of FMD cases in Europe in the late 1960s. Despite successful vaccination, nucleotide sequence analyses provided evidence that a few disease outbreaks in Europe in the 1980s were caused by vaccines, presumably due to insufficient inactivation of the virus or due to viruses that escaped from vaccine factories (Sobrino et al., 2001). Unfortunately, despite remarkable progress, no effective synthetic

1968; Pereira, 1981). Viruses of a different serotype do not manifest crossprotection. In addition, many subtypes within a serotype, which evoke partially cross-protective immune responses, were identified by classical immunological techniques. Use of monoclonal antibodies

The FMD outbreak must call into question whether the existing health policies in the EU are still adequate in the context of a highly competitive and a global economy

unwanted outcome after recovery of ruminants from an acute infection-some FMDV genes can reach an evolutionary rate of $7 \times 10^{-2}$ mutations per nucleotide site per year (Domingo et al., 2001). Despite the fact that negative selection tones down the evolutionary potential, it should not come as a surprise that PanAsia viruses did not exist fifteen years ago when the first FMDV phylogenies were produced. Now these new swarms are displacing other pre-existing viruses (Knowles et al., 2001).

Genetic heterogeneity and diversification may result in amino acid substitutions in virtually any FMDV protein, but particularly in exposed surface loops of capsid proteins, which critically affect the antigenic properties of the virus. On the basis of cross-protection studies with convalescent and vaccinated animals, seven serotypes documented that FMDV populations are composed of a continuum of antigenic variants, with amino acid substitutions affecting virtually all of the several antigenic sites which have been defined on viral particles (Mateu, 1995).

For successful protection, vaccines must match the antigenic properties of the circulating viruses, which is made more difficult by the antigenic heterogeneity among co-circulating viruses within individual animals. Protection requires that vaccines must include multiple virusspecific B-cell and T-cell epitopes, inducing an ample repertoire of neutralising antibodies in order to minimise selection of variant viruses which arise during replication (Sobrino et al., 2001). This requirement was partially fulfilled by whole-virus inactivated polyvalent vaccines, classically employed for FMD control (Bachrach, vaccines, which do not require handling of live virus, were available. This is partly due to low efficiency in the production of non-infectious, entire particles, such as empty capsids or viruses with genomic deletions. Also, synthetic peptides conferred only partial protection in natural hosts because the multiple-epitope rule was often not followed. Given the commercial advantages of a FMD-free continent, with all world markets open to trade of meat and meat products, the EU adopted a non-vaccination policy in 1991, which has rendered European farm animals fully susceptible to the virus. Indeed, in light of an increasingly global economy, the nonvaccination decision was risky.

The epidemiology of FMD is the result of a large number of interconnected factors related to the virus itself, its hosts, and the environment. A number of factors may affect virus virulence-number and

Given the commercial advantages of a FMD-free continent, the EU adopted a non-vaccination policy in 1991

severity of lesions, duration of the infectious cycle, organs affected, etc.- - the host species preferentially infected, the number of particles excreted per infected animal, particle stability in various microenvironments and 


\section{viewpoint}

chances of long-term persistence. The host may modulate FMDV multiplication, antigenicity and spread, depending on the host species, nutritional and immunological status, population density, animal move-

of necessary knowledge to contain further outbreaks. Unfortunately, basic research on FMDV in the EU was severely curtailed over the last decade and teams of experts have 'moved' to other viruses.

\section{In a global economy with open markets and frequent exchanges of goods over long distances, the completely unprotected, crowded farm animal population in Europe provided an ideal scenario for the re-emergence of FMD}

ments, contacts between animals of different species, and the presence of wild animals capable of sustaining virus replication. The environment can provide barriers to virus dissemination, such as mountain ranges, seas, deserts, areas free of susceptible hosts, or can promote virus transmission when appropriate conditions prevail. Low temperature, high humidity and weak sunshine favour airborne transmission of virus particles. Contaminated animal products, nonsusceptible carrier animals, agricultural tools, people, vehicles, or arthropod vectors can contribute to the mechanical transmission of FMDV (Bachrach, 1968; Pereira, 1981). The FMDV mutant swarms, their multiple hosts and the complexity of environmental features provide a set of variables which renders FMD outbreaks and their evolution essentially unpredictable and uncontrollable.

The emergence and re-emergence of infectious diseases is influenced by pathogen adaptability in connection with environmental, ecological, technological and sociological factors (Lederberg et al., 1992). In a global economy with open markets and frequent exchanges of goods over long distances, the completely unprotected, crowded farm animal population in Europe provided an ideal scenario for the re-emergence of FMD. These circumstances were probably not fully appreciated at the time at which the non-vaccination policy was adopted. Yet it has long been known that the most dramatic effects of FMDV are felt in previously virus-free areas or when a new serotype enters an enzootic area (Pereira, 1981). The pathogenic characteristics of the PanAsia FMDV, together with the full susceptibility of animals, is therefore contributing to the severity of the ongoing European outbreak.

Regarding the current situation, we think that basic science can provide a lot

Yet we know little about FMDV pathogenesis, such as the events in early infection, the source of the high viral loads during the viremic phase of disease or the basis of cell and tissue tropism, or about the type of immune response needed to confer full protection. The paralysis of FMDV research has also impeded further progress in the development of synthetic vaccines despite huge investments made only one decade ago. From what we have learned from work on FMDV and other viruses, it is therefore justified to re-launch research on the development of vaccines based on replication-defective viruses or cocktails of synthetic antigens with markers to distin-

\section{Basic research on FMDV in the EU was severely curtailed over the last decade and teams of experts have 'moved' to other viruses}

guish vaccinated from infected animals. Such distinction should be feasible with recently available procedures (reviewed in Sobrino et al., 2001).

The re-emergence of FMD in Europe should drive a serious debate on two fronts. First, we need a scientific assessment of the advantages and disadvantages of a vaccination versus a non-vaccination policy under an increasingly global economy. Secondly, the EU must evaluate the risks for economy and society of facing the consequences-massive slaughtering of animals and dangers for the wild fauna-of a highly virulent disease that affects non-immune animal populations. The outbreak in the UK is indeed not a unique, unrepeatable event (Lederberg et al., 1992). Its occurrence has raised questions not only about agricultural policies in the EU, but also about the adequacy of measures implemented to cope with the emergence and re-emergence of human, animal or plant diseases. With the current surveillance policies and strategies, how long would it take to report to health authorities in Brussels a case of say, fatal meningococcal meningitis or African Swine Fever occurring at the fringes of the EU? And what measures should be taken to prevent epidemics in the first place?

\section{References}

Acharya, R. et al. (1989) The three-dimensional structure of foot-and-mouth disease virus at 2.9 Å resolution. Nature, 337, 709-716.

Bachrach, H.L. (1968) Foot-and-mouth disease virus. Annu. Rev. Microbiol., 22, 201-244.

Domingo, E., Biebricher, C., Eigen, M. and Holland, J.J. (2001) Quasispecies and RNA Virus Evolution: Principles and Consequences. Landes Bioscience, Austin, TX.

Knowles, N.J. et al. (2001) Outbreak of foot-andmouth disease virus serotype $\mathrm{O}$ in the UK caused by a pandemic strain. Vet. Rec., 148, 258-259.

Lederberg, J., Shope, R.E. and Oaks, S.C. (1992) Emerging Infections. Microbial Threats to Health in The United States. National Academy Press, Washington, DC.

Mateu, M.G. (1995) Antibody recognition of picornaviruses and escape from neutralization: a structural view. Virus Res., 38, 1-24.

Pereira, H.G. (1981) Foot-and-mouth disease virus. In Gibbs, R.P.G. (ed.), Virus Diseases of Food Animals. Vol. 2. Academic Press, New York, NY, pp. 333-363.

Sobrino, F. et al. (2001) Foot-and-mouth disease virus: a long known virus, but a current threat. Vet. Res., 32, 1-30.
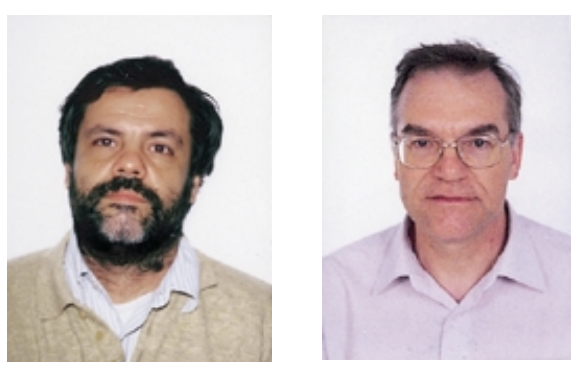

Francisco Sobrino and Esteban Domingo are at the Centro de Biología Molecular 'Severo Ochoa' of the Universidad Autónoma de Madrid, Cantoblanco and at CISA-INIA, Valdeolmos in Spain.

E-mail: fsobrino@cnb.uam.es or

edomingo@cbm.uam.es

DOI: $10.1093 / \mathrm{embo-reports/kve122}$ 